\title{
Sleep apnoea occurring only in REM (possible relevance of the case for some cot deaths)
}

\author{
M. D. BEARY \\ M.A., M.B., B.Chir. \\ M. B. CRUTCHFIELD \\ B.Sc.
}

\author{
M. K. Mintram \\ B.A., MPhil.
}

\author{
A. H. CRISP \\ M.D., D.Sc., F.R.C.P., F.R.C.Psych.
}

Academic Department of Psychiatry, St. George's Hospital Medical School, Tooting, London SW17 ORE.

\begin{abstract}
Summary
A case of sleep apnoea occurring only within REM sleep in a 57-year-old man is described. It was successfully treated with clomipramine for a period. The patient probably had persistent phrenic nerve damage which accounted for, or contributed to, the phenomenon. It is suggested that this mechanism may operate in some cot deaths.
\end{abstract}

\section{Introduction}

A case is described of sleep apnoea in rapid eye movement (REM) sleep only, a relatively rare phenomenon, which responded to treatment. It is suggested that this response and the unusual pathology revealed may shed some light on mechanisms leading to some cot deaths, the sudden infant death syndrome (SIDS).

\section{Patient}

A 57-year-old married club organizer has a 5year history of falling asleep up to 10 times during the day, with hypnogogic hallucinations and disturbed night-time sleep. His wife has noticed that he appears not to breathe for long periods of the night.

\section{Psychodynamic factors}

$\mathrm{He}$ is a conscientious, outwardly cheerful and composed moral man who was partially sighted from childhood. He was sexually assaulted at the age of 10 years and has had sexual ruminations ever since. $\mathrm{He}$ is long married but infertile and has current sexual problems. He has lost five close relatives over the last seven years.

\section{Physical factors}

When 16 years old, he was knocked out in a landmine explosion. Both arms were fractured and he had abdominal and chest injuries. At 21 years he suffered from pulmonary tuberculosis. He was treated with a phrenic nerve crush and with surgical pneumothoraces which were corrected in 1949. He has since suffered from chronic bronchitis each winter. Physical examination revealed the expected surgical and traumatic scars. His chest was abnormal being pigeon-breasted with extremely reduced expansion. There were rhonchi bilaterally. Cardiovascular system, abdomen and central nervous systems-normal. Chest $\mathrm{X}$-ray revealed extensive pleural calcification and no phrenic nerve paralysis.

\section{Polygraphic sleep recordings}

Twenty-four hour recording revealed five short periods of sleep without REM onset in the day. Four nights were recorded. Two baseline nights showed normal sleep staging fragmented by wakefulness from stage 2, but not for REM. He had 35 (first night) and 55 (second night) periods of apnoea (Fig. 1) in REM only (mean $=29 \mathrm{sec}$, range $14-50$ $\mathrm{sec}$ ). In one 12-min period, $5 \mathrm{~min} 9 \mathrm{sec}$ were spent apnoeic. There was virtually no arousal even after 50-sec apnoeas.

On night $3,30 \mathrm{mg}$ flurazepam had no effect on his apnoeas. On night 4 , clomipramine $150 \mathrm{mg}$ produced grossly atypical sleep staging with high muscle tension throughout (the patient woke and vomited after $92 \mathrm{~min}$ and returned to sleep one hour later). No sleep apnoeas were observed during the recording.

\section{Treatment and progress}

The only treatment offered was clomipramine $25 \mathrm{mg}$ at night. It was decided that the extent and chronicity of the psychopathology was such that psychotherapy was contra-indicated. After 2 weeks he had experienced no daytime napping, had slept well at night and felt physically fitter than for many years. Thereafter he began to relapse, but 6 months later he remains substantially better than before. 


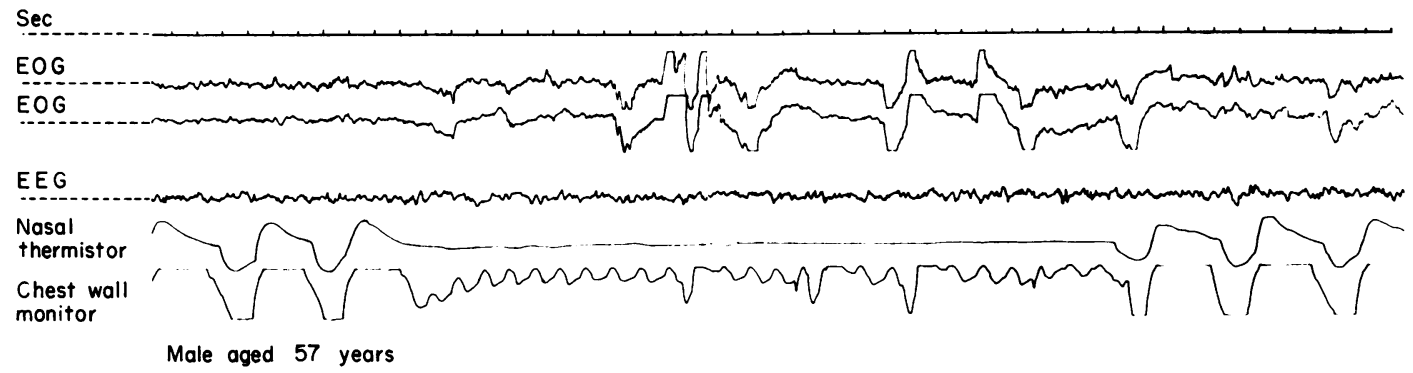

FIG. 1. A 30-sec period of apnoea in REM as demonstrated by nasal thermistor and chest wall monitor (this shows only cardiac impulses plus occasional diminished respiratory effort). EOG=electro-ophthalmogram; EEG=electro-encephalogram.

\section{Discussion}

Sullivan and Issa (1980) have shown that patients with sleep apnoea tend to have longer periods of apnoea during REM than non-REM (NREM). Orem (1980) has demonstrated fractionation of diaphragmatic activity in cats. During REM sleep, Phillipson et al. (1980) have demonstrated that fragmentation of sleep in dogs will elevate arousal thresholds to hypercapnoea and hypoxia. They postulate this as the first stage of hypersomnia and sleep apnoea.

The authors believe that their patient's impaired chest function together with his iatrogenically damaged phrenic nerve may have created a situation where in REM sleep the paralysis of the intercostal muscles and the fractionation of diaphragmatic activity has led to central elevation of arousal thresholds and attacks of apnoea. The absence of chest wall/diaphragmatic movement on the recordings associated with zero airflow at the nasal thermistor, suggested that in REM either the nerve impulses cannot pass the phrenic nerve damage or, in addition as they suspect because of the lack of arousal after apnoea, there has been central elevation of arousal thresholds. They conclude that their patient's daytime sleepiness is mainly a reaction to this disturbance, rather than related to his sexual psychopathology as is sometimes the case (Crisp, 1979).

The progression from phrenic nerve damage, as $\infty$ in birth injury, to elevation of central arousal thresholds and apnoea, postulated as characterizing this case, is perhaps a model for the mechanism of some cot deaths.

In their laboratory, the authors have shown that clomipramine greatly increases muscle tension during sleep both in REM and NREM tending to mask the former which, however, persists (Crisp, 1980). This probable central action of the drug ma\& have contributed importantly to its special effective ness in the management of this case as well as making it a useful agent for the treatment of sleep apnoea ip general.

\section{References}

CRISP, A.H. (1979) Sexual pathology in the psychiatric clinic. British Journal of Clinical Practice, 5, 3.

CrisP, A.H. (1980) Sleep, activity, nutrition and mood. British Journal of Psychiatry, 137, 1.

OREM, J. (1980) Neuronal mechanisms of respiration in REM sleep. Sleep, 3, 251.

Phillipson, E.A., Bowes, G., Sullivan, C.E. \& Woolf, G.M. (1980) The influence of sleep fragmentation on arousal and ventilatory responses to respiratory stimuli. Sleep, 3, 281.

Sullivan, C.E. \& Issa, F.G. (1980) Pathophysiological mechanisms in obstructive sleep apnoea. Sleep, 3, 235. 